Revista de Filosofía y Teoría Política, n. ${ }^{\circ}$ 49, 2018, e024, ISSN 2314-2553

Universidad Nacional de La Plata.

Facultad de Humanidades y Ciencias de la Educación.

Departamento de Filosofía

\title{
¿Academicismo o liderazgo continental? Algunos contrastes en la definición de la Reforma Universitaria
}

\author{
Alejandra Mailhe \\ Universidad Nacional de La Plata, CONICET, Argentina \\ amailhe@fahce.unlp.edu.ar
}

Cita sugerida: Mailhe, A.(2018). ¿Academicismo o liderazgo continental? Algunos contrastes en la definición de la Reforma Universitaria. Revista de Filosofía y Teoría Política, (49), e024. https:/doi.org/10.24215/23142553e024 


\title{
¿Academicismo o liderazgo continental? Algunos contrastes en la definición de la Reforma Universitaria
}

\author{
Alejandra Mailhe \\ Universidad Nacional de La Plata, CONICET, Argentina \\ amailhe@fahce.unlp.edu.ar
}

\section{Resumen:}

Este trabajo reflexiona sobre dos perfiles intelectuales contrastantes: el del profesor argentino Ernesto Quesada, y el del intelectual mexicano José Vasconcelos, de visita en Argentina en 1922. Tal como veremos, ambos definen la Reforma Universitaria de forma contrastante, pocos años después de 1918: mientras Quesada aboga por concebir la Reforma como una experiencia clave para avanzar en la profesionalización de la docencia y la investigación académicas, autonomizando estas prácticas respecto de la política, Vasconcelos resiste la especialización disciplinar y defiende la construcción de liderzgos políticos continentales, en el seno del movimiento reformista, en sintonía con la experiencia de la Revolución Mexicana. Asimismo, el trabajo atiende a algunos puntos de contacto entre Vasconcelos y el argentino Ricardo Rojas, quienes convergen y se distancian ideológicamente en la valoración de la Reforma, en el arco que va de los años veinte a los treinta.

Palabras ClaVe: Ernesto Quesada, José Vasconcelos, Ricardo Rojas, Movimiento reformista.

\section{ABstraCt:}

This article reflects on two contrasting intellectual profiles: the Argentinian university lecturer Ernesto Quesada and the Mexican intellectual José Vasconcelos, who visited Argentina in 1922. As we shall see, both define the University Reform in a contrasting way a few years after 1918. On the one hand, Quesada advocates conceiving the Reform as a key experience in order to make advances in the professionalisation of university teaching and research, autonomising these practices from politics. On the other hand, Vasconcelos resists disciplinary specialisation and defends the construction of continental political leaderships, within the reformist movement, in line with the Mexican Revolution experience. Likewise, this article addresses certain points of contact between Vasconcelos and the Argentinian Ricardo Rojas who ideologically converge and also distance themselves from each other on their assessment of the Reform in the years that spanned the 1920s to 1930s.

KEYWORDS: Ernesto Quesada, José Vasconcelos, Ricardo Rojas, Reformist movement.

La Reforma Universitaria constituye uno de los movimientos de transformación más relevantes en la historia de las instituciones educativas superiores de América Latina. Entre otros cambios, democratiza el gobierno de las universidades, y abre más francamente la posibilidad, a los sectores medios, de acceder a un título universitario. Sus postulados promueven el co-gobiernos de las universidades (con participación estudiantil y de profesores en los órganos de la Universidad), la autonomía universitaria, la libertad de cátedra y la extensión universitaria. Su impacto se extiende por las universidades de Argentina y de América Latina, como Perú, Chile y México.

Partiendo de este marco muy general, este trabajo reflexiona sobre dos perfiles intelectuales contrastantes: el del profesor argentino Enresto Quesada, y el del intelectual mexicano José Vasconcelos, de visita en Argentina en 1922. Tal como veremos, ambos definen la Reformar Universitaria de forma contrastante, pocos años después de 1918: mientras Quesada aboga por concebir la Reforma como una experiencia clave para avanzar en la dedicación exclusiva a la docencia y la investigación académicas, autonomizando estas prácticas respecto de la política, Vasconcelos resiste la especialización disciplinar y defiende la construcción de liderazgos políticos continentales, en el seno del movimiento reformista, en sintonía con la experiencia de la Revolución Mexicana. Asimismo, el trabajo atiende a algunos puntos de contacto entre Vasconcelos y el argentino Ricardo Rojas, quienes convergen y se distancian ideológicamente en la valoración de la Reforma, en el arco que va de los años veinte a los treinta. 


\section{LOS LÍMITES DEL REFORMISMO ACADÉMICO: ERNESTO QUESADA}

Hijo de Vicente Quesada - un importante diplomático y abogado-, Ernesto Quesada es educado en su primera infancia en diferentes países (Bolivia, Brasil, EE.UU., México, España, Alemania y el Vaticano, entre otros), siguiendo los cargos diplomaticos de su padre. Luego estudia en las universidades de París, Dresde, Leipzig y Berlín, y egresa de la Facultad de Derecho de la UBA en 1882. Tal como ha probado Buchbinder (2012), este itinerario intelectual prefigura en gran medida su inclinación por la dedicación exclusiva a la docencia y a la investigación académicas.

Por otro lado, Quesada inicia tarde su breve carrera docente, en 1905, a los 50 años, como primer profesor titular de la cátedra de Sociología de la Facultad de Filosofía y Letras de la UBA, la primera cátedra de sociología del país. En 1907 lo nombran además profesor a cargo de "Economía política" en la Facultad de Ciencias Jurídicas y Sociales de la UNLP, retirándose de la docencia en 1922

En sus clases publicadas y en sus conferencias, es posible entrever en qué medida Quesada busca articular la profesionalización de la sociología (y de la docencia universitaria en general) con el ejercicio de un liderazgo reformista moderado, lo que no deja de implicar la presencia de algunas contradicciones significativas. En efecto, si bien Quesada despliega sus actividades como historiador, sociólogo, profesor universitario, abogado, juez y germanista, se ve a sí mismo sobre todo como un hombre de ciencia, consagrado a la vida académica, lo que supone un importante esfuerzo por implantar la profesionalización de la investigación y la docencia universitarias, con el consecuente abandono de las funciones públicas tradicionales de la elite. En este sentido, tal como señalan Bergel (2008) y Buchbinder (2012), Quesada se esfuerza por consolidar formas más modernas de legitimación: frente al desgaste de las vías más tradicionales, propias del viejo patriarcado, porta un capiltal simbólico fundado en la consagración casi exclusiva a la tarea académica. Ese empeño por conquistar la autonomía académica respecto de la política se palpa, por ejemplo, en la elaboracion de extensos y minuciosos textos eruditos, cargados de bibliografía crítica (tal como ocurre con el curso que dicta en 1921 sobre la "sociología spengleriana"), en el cambio constante de sus programas de estudio, en al introducción de novedosos objetos de investigación (como el marxismo, el feminismo o el propio relativismo spengleriano), y en la fuerza con que impulsa a los estudiantes universitarios a formular nuevas críticas a los modelos teóricos centrales, poniéndolos a prueba frente a los problemas argentinos y latinoamericanos. A estos gestos se suma su adhesión juvenil al positivismo, pero desde una perspectiva crítica del biologicismo (que, al menos en parte, facilita su incursión posterior en la filosofía de Spengler), y su temprano interés por las periferias, tangible en sus libros de viaje por Rusia y Oriente. ${ }^{1}$ Estos elementos convierten a Quesada en un mediador intelectual privilegiado para introducir las tesis de Spengler sobre "la decadencia de Occidente" en América Latina.

Como parte de ese perfil modernizador, Quesada defiende la autonomía universitaria, en sintonía con los principios de la Reforma de 1918. ${ }^{2}$ Bajo el modelo prusiano de Universidad, aboga por la dedicacion exclusiva a la investigación y la docencia. Sin embargo, tal como advierte Pereira (2008), el campo intelectual argentino -dominado por políticos y profesionales liberales, más que por científicos- todavía es hostil a ese nuevo modelo. En parte como resultado de su imposibilidad de realizar su ideal de profesionalización, Quesada renuncia a sus cátedras en 1922, se autoexilia en el exterior, y termina donando su biblioteca al estado prusiano y no a la UBA, como hubiese querido (según se deja entrever en la conferencia que dicta en 1921 en la UBA, con la que se cierra la edición de La sociología spengleriana).

El germanismo de Quesada se traduce en su admiración por el modelo prusiano de ilustración positiva y humanista, en la adopción de una perspectiva pro-alemana durante la Primera Guerra Mundial, y en la difusión de la "sociología" de Spengler (en parte, gracias a la mediación de su segunda mujer, la periodista y escritora alemana Leonore Deiters).

Desde el punto de vista político, Quesada defiende la participación activa del Estado en la mejora de las condiciones socioculturales de la población, desde una perspectiva reformista que, en dfinitiva, busca 
evitar cualquer desborde revolucionario. ${ }^{3}$ Ese reformismo, ya visible en los textos producidos torno al novecientos, moldea el gesto paternalista con que, en los años veinte, se propone guiar el "despertar indígena", que preanuncia al corregir el pronóstico de Spengler.

Desde sus textos tempranos, producidos al calor de las primeras protestas estudiantiles a inicios del siglo XX, Quesada se manifiesta como un reformisa que aboga por la autonomía de la Universidad respecto de la política, y en consecuencia asume la defensa de la dedicación plena a la docencia y la investigación, en claro contrasto con respecto al magisterio militante y resistente a la especialización, de figuras como José Vasconcelos, que luego de la experiencia de 1918 se consolida como "Maestro de la Juventud" a nivel continental.

Ya en 1906, en el folleto La crisis universitaria (que responde a la temprana inquietud estudiantil desatada, entre 1904 y 1906, en las facultades de Derecho y Ciencias Sociales, y en la de Ciencias Médicas), Quesada defiende el ideario reformista de los estudiantes que se rebelan contra el orden universitario dominante. Considera que, frente a la protesta estudiantil, las autoridades deben introducir modificaciones que preserven el apostolado universitario, para evitar la violencia de la rebelión, pues cuando los cerebros estudiantiles son "demasiado ardorosos e impacientes", crece el conflicto y puede "peligrar la vida misma de la institución" (Quesada, 1906, p. 19). Ya por entonces -en plena discusión de diversos proyectos de reforma, promovidos por diputados en el Congreso-, Quesada adhiere a la formación de una alta cultura científica, independientemente de las necesidades prácticas de formación pro pane lucrando.

Ese punto de vista se profundiza en el contexto de la Reforma de 1918. En efecto, en El ideal universitario (el folleto que recoge la conferencia dada en la Facultad de Filosofía y Letras de la UBA, el 24 de agosto de 1918, ya desatada la rebelión reformista), Quesada se propone reflexionar sobre la mejor orientación de la educación superior, teniendo en cuenta la crisis que condujo recientemente a la clausura momentánea de la Universidad de Córdoba, y su repercusión en otras universidades de país. Puesto a historizar el modelo didáctico vigente en las universidades argentinas, en un gesto que vuelve a subrayar su auto-identificación con el papel de docente-investigador (en la medida en que convierte la conferencia reformista en una clase de historia más que en una experiencia de comunión política), subraya en qué medida el model implantado en el país responde a la vieja escolástica jesuítica, luego recreada en la universidad napoleónica de Francia. Ese modelo, fundado en el estudio de disciplinas fijas organizadas en años sucesivos, incluyendo además la asistencia obligatoria a clase, y la rendición de exámenes parciales y finales, es puesta en contraste con respecto al modelo inglés y norteamericano (donde la universidad se centra en la enseñanza tutorial y directa del maestro, y en la estructura de los colleges), y es con respecto al modelo prusiano, el ideal para Quesada, porque promueve la ciencia pura y la investigación científica, y por ende la dedicación exclusiva de los docentes a la enseñanza y la investigación, evitando las materias escolares con planes de estudio obligatorios tanto como los exámenes parciales como "la pedagogía de la Compañía de Jesús"). Para Quesada, el "concepto cesariasta de la enseñanza", presente en el modelo jesuítico aun vigente en el país, " constituye [...] un lecho de Procusto para la mentalidad de la nación entera" (Quesada, 1918, p. 8), porque implica equiparar al estudiante universitario con un menor incapaz que debe ser tutelado, "e impide que el carácter personal se revele" (Quesada, 1918: p. 26). Quesada cree que las universidades deben alcanzar la autonomía económica para garantizar así el auto-gobierno; propone la elección anual de rectores sin mecanismos de re-elección, para preservar así la democracia; que los docentes tengan absoluta libertad de cátedra, siempre y cuando demuestren apego a la investigación; exige que los cursos sean abiertos y no obligatorios, y defiende la concurrencia mixta de varones y mujeres. Pero también, en un gesto que conserva un fuerte componente elitista, se opone a los concursos docentes, argumentando que los profesores más talentosos (imprescindibles para la formación personal de los mejores discípulos) se niegan a semejantes formas de mesura. Además, concibe la Universidad como regida por un criterio de "libre mercado", al estimular la competencia entre los docentes por la obtención del mayor número de alumnos en sus cursos, como condicionante del salario (en la medida en que los cursos continúan siendo pagos). 
Para Quesada, la Universidad (sacralizada como un templo del conocimiento, que incluso garantiza la preservación de los valores espirituales frente a la crisis identitaria desatada en el marco del aluvión inmigratorio ${ }^{4}$ exige la consagración plena a la docencia y la investigación, en desmedro de cualquier participación política, incluso para evitar conflictos innecesarios entre docentes y alumnos: "sería quizá de anhelar que los enseñantes universitarios se abstuvieran de participar en la vida política militante para no verse expuestos a extremos [...]. El hombre que dedica su vida a la enseñanza universitaria con verdadera vocación, se coloca fuera de las tentaciones de la política activa: cuando toma parte de ésta es porque aquella vocación no ha sido firme" (Quesada, 1918, p. 25). En este sentido, en los límites conservadores del reformismo, su defensa de la profesionalización científica, en desmedro de la dependencia respecto de la política, coloca a Quesada en las antípodas del Magisterio de la Juventud ejercido -como veremos- por figuras como José Vasconcelos.

Sin embargo, el curso universitario sobre La decadencia de Occidente del filósofo alemán Oswald Spengler, dictado en 1921 en la UBA y la UNLP, puede leerse también como parte de un movimiento de autolegitimación del docente universitario como intelectual reformista, entiendiéndolo a éste como una figura capas de intervenir en los debates sociales y políticos, pero apelando a la defensa de su especificidad profesional. En este caso por ejemplo, gracias a su apropiación crítica del relativismo cultural spengleriano, Quesada logra formular un americanismo académico, al tiempo que hace converger sus aspiraciones de especialización universitaria con la demanda de compromiso político propia del reformismo.

En la figura y en la obra de Spengler, Quesada parece encontrar un modo de acompañar el compromiso reformista y la adhesión creciente al espiritualismo, al relativismo cultural y al americanismo, desde una vertiente de la filosofía alemana -privilegiada desde su germanismo-, pero a la vez disidente respecto del neokantismo hegemónico en el medio local, ${ }^{5}$ más inclinado en favor de reivindicar, en términos arielistas, el legado "latino" o incluso hispano-católico de la identidad. En este cierre de su carrera académica, la difusión de Spengler le permite a Quesada proyectarse como una figura "política", interpelando a un lectorado reformista más amplio que el acotado círculo de especialistas, pero sin abandonar su especificidad disciplinar, incluso probando el alcance de su influencia como "Maestro" del reformismo a nivel continental, aunque desde un lugar más modesto que el de figuras como José Vasconcelos.

Luego de la lectura del primer tomo de La decadencia... en su edición alemana ${ }^{6}$-gracias a la mediación de su segunda esposa Leonore Deiters, y de un familiar de ella en Alemania-, Quesada accede a una amplia bibliografía crítica sobre Spengler, e incluso estableces un vínculo personal con él, gracias al intercambio de una abultada correspondencia entre ambos, y a la realización de varias entrevistas personales, en los tres viajes a Europa, que concreta antes de instalarse definitivamente en Suiza a fines de la década del veinte. ${ }^{7}$

Su lectura de la obra de Spengler -que lo consagra como el primer introductor de la obra del alemán en Argentina y en América Latina- se plasma en varias tesituras discursivas diferentes, que van desde el grueso volumen del curso universitario de 1921, a las conferencias, más académicas en el caso de las que dicta en las universidades nacionales de Córdoba y de La Plata en $1923,{ }^{8}$ o más "políticas" en el caso de la que presenta en La Paz en 1926, frente a un público que incluye a la elite dirigente y al estudiantado reformista de Bolivia.

En su curso de 1921, Quesada revisa minuciosamente tanto los argumentos del alemán como las polémicas que ese libro desata dentro y fuera de Alemania. ${ }^{9}$ Allí el autor no se limita a reseñar las críticas ajenas, sino que también somete el libro a su propia discusión, partiendo de un punto de vista americanista, que le exige poner en juego una férrea defensa de la arqueología del mundo precolombino. En efecto, en ese curso Quesada le señala al autor de La decadencia..., la necesidad de estudiar mejor el caso americano, considerando especialmente las civilizaciones previas a la conquista, tanto para demostrar con mayor rigor la hipótesis sobre el carácter monádico de las culturas, como para corregir la predicción sobre el nuevo ciclo cultural, que para Quesada no será eslavo -como supone Spengler a la luz de la Revolución Rusa-, sino americano, y especialmente indígena. 
Quesada, que se dedica al estudio de las sociedades precolombinas en su curso de $1917,{ }^{10}$ insiste en esta crítica a Spengler a lo largo de todos sus trabajos sobre La decadencia.... Así por ejemplo, en la conferencia que da en la UNLP de 1923 (es decir, cuando ya ha accedido a la lectura del segundo tomo del libro de Spengler), advierte el tratamiento desparejo que le da el alemán a los diversos ciclos culturales, deteniéndose mucho en la cultura árabe, y en cambio desconsiderando la azteca, a la cual apenas nombra como ejemplo "del final violento de una cultura" (Quesada, 1923, p. 21)

Esta crítica al residuo eurocéntrico en la mirada de Spengler, se articula con el esfuerzo, de parte de Quesada, por corregir el pronóstico del alemán, para quien -como dijimos- el nuevo ciclo será eslavo, tal como puede apreciarse en el campesinado ruso, no tocado por la decadencia occidental, y activado por la Revolución. ${ }^{11}$ En cambio, para Quesada el nuevo ciclo será americano, y especialmente indígena. Si en el curso de 1921 ya se postulan sus hipótesis indigenistas, en sintonía con sus discursos más francamente centrados en la Reforma (como El ideal universitario, o su conferencia de despedida de la UBA en 1921, editado en La sociología relativista spengleriana), su esfuerzo por sostener un delicado equilibrio entre profesionalización y reconocimiento político (en sentido amplio) se percibe en su viaje a La Paz en 1926: la conferencia que dicta allí, en la Universidad Mayor de San Andrés, puede leerse en sintonía con su esfuerzo por compatibilizar especialización académica e intervención reformista en la esfera pública. Tanto el contenido de ese discurso, como la recepción del público paceño (parciamente registrada en la edición de 1926), e incluso el propio itinerario del viaje de Quesada por el área andina (tensionado entre el aprendizaje arqueológico y las intervenciones públicas)refuerzan esa ambivalencia entre el academicismo y el liderazgo reformista (liderazgo que Quesada busca resolver desde la especificidad de su métier).

Creemos que la vehemencia con que Quesada le insiste a Spengler en su necesidad de ahondar en las culturas americanas obedece a varias motivaciones diferentes. Entre otras cosas, porque Quesada valoriza una consagración exclusiva a la investigación, que incluye necesariamente el ejercicio de la crítica. No casualmente, en su curso de 1921, en plena sintonía con su concepción de la Reforma como transformación académica, defiende el cuestionamiento de los modelos teóricos como una parte imprescindible para la producción de conocimiento en la Universidad, e incita a los alumnos a colaborar con la obra del autor de La decadencia..., poniendo a prueba sus hipótesis, al aplicarlas al mundo precolombino y a la historia americana en general, pues "para el estudiante hispanoamericano, lo más interesante en el estudio de esta nueva doctrina sociológica es que podrán colaborar activamente en ella, llenando el vacío relativo a las culturas pre y postcolombinas, en lo cual el libro de Spengler es absolutamente deficiente" (Quesada, 1921, p. 20). Y agrega que, al someter la teoría de Spengler a esta prueba, podrán "confirmar, refutar o modificar" las conclusiones del libro (Quesada, 1921, p. 27). ${ }^{12}$ En este sentido, las críticas de Quesada a Spengler pueden leerse como parte de una rebelión contra cualquier forma pasiva de recepción: Quesada imagina una discusión con el centro casi en paridad de condiciones, o incluso desde una posición de cierta superioridad, dado el conocimiento propio sobre el mundo americano. Y esa afirmación identitaria, desde la especificidad del saber académico, también responde al clima ideológico de la Reforma Universitaria.

Incluso en la construcción de la imagen de Spengler se deja entrever su ideal de un liderazgo reformista no tocado por la esperiencia política. Así por ejemplo, en su curso de 1921, Quesada presenta a Spengler apelando a una figuración clisé, propia del espiritualismo: el alemán aparece como un asceta reconcentrado en sus meditaciones, "por completo apartado del mundo, entregado a sus intesivas investigaciones sociológicas [...], casi secuenstrándose de todos y de todo, tanto que vive, como un monje laico, en un par de habitaciones llenas de libros, y no recibe a casi nadie y lo hace por poco tiempo" (Quesada, 1921, p. 22). Esa cita le permite a Quesada no solo exhibir la exclusividad de su vínculo con Spengler, sino también a subrayar la compatibilidad entre la filosofía spengleriana y los valores del reformismo espiritualista local (según su versión autonomizante), espejando en Spengler el modelo del Maestro "Próspero" en el Ariel (1901) de José E. Rodó, fundado en el desinterés material y en el cultivo de la espiritualidad, aunque ahora pasado por el tamiz de la consagración a la investigación. ${ }^{13}$ 
En definitiva, en los trabajos de Quesada sobre Spengler es posible entrever varios principios de su reformismo profesionalizante y, al mismo tiempo, reconocer su esfuerzo por articular la autonomización de la práctica académica y la intervención (mediada) en el campo de la política, al tiempo que, además, la recepción de Spengler supone apropiarse de un modelo filosófico cargado de connotaciones reaccionarias (que se harán más visibles en los años treinta, con la publicación del ensato Jahre de Entscheidung [Años decisivos], y amalgamarlo con el progresismo propio de la adhesión de Quesada a la Reforma Universitaria y a su versión del americanismo. La mediación ejercida por Quesada supone entonces la interpelación directa tanto del alemán como del público reformsita local, y la torsión del análisis y del pronóstico de Spengler, para completar y corregir ese modelo a partir de la visibilización de América Latina.

\section{LA CONSTRUCCIÓN DE UN LIDERAZGO REFORMISTA CONTINENTAL: JOSÉ VASCONCELOS}

Licenciado en Derecho en 1907 y miembro fundador del Ateneo de la Juventud Mexicana, Vasconcelos se manifiesta tempranamente como crítico del positivismo hegemónico en el Porfiriato, comprendiendo los ideales ateneístas de defensa del espiritualismo y de los valores éticos y estéticos hispanoamericamos. Partidario de la Revolución Mexicana desde sus inicios (al apoyar el movimiento anti-reeleccionista de Francisco Madero, con quien comparte además sus afinidades teosóficas), se exilia en EE.UU. por su posición crítica frente a Venustiano Carranza. Caído ese gobierno, regresa a México para ser nombrado Rector de la Universidad Nacional entre junio de 1920 y octubre de 1921. Durante su gestión, impulsa proyectos educativos destinados a los sectores populares, y alienta la realizaciión del "Primer congreso internacional de estudiantes", una instancia clave en al circulacioón transnacional de ideas y de figuras en el marco del reformismo continental.

Entre octubre de 1921 y julio de 1924 se desempeña como Secretario de Educación Pública, ${ }^{14}$ iniciando un ambicioso plan de instrucción popular que realiza, en gran parte, el modelo inclusivo pergeñado previamente por Manuel Gamio en Forjando patria (1916). En este sentido, Vasconcelos promueve la alfabetización masiva, la educación de la población rural e indígena, la creación de bibliotecas populares, la edición de libros baratos y la difusión del arte, apoyando especialmente el potencial pedagógico del muralismo mexicano y de la arquitectura. Además, pone en marcha un amplio plan de intercambio estudiantil con otros países latinoamericanos, y protege a intelectuales perseguidos como el peruano Victor Haya de la Torre. Estas prácticas modelan la internvención cultural del Estado durante años, tanto en México como en el resto del continente, y colaboran en la consagracióm de Vasconcelos como "Maestro de la Juventud", en el marco de la Reforma Universitaria.

El vínculo del autor de La raza cósmica con los jóvenes reformistas argentinos se vertebra en base a dos hitos significativos: el viaje de la delegación argentina a México en 1921, y el viaje de Vasconcelos a la Argentina en 1922. Gracias a ambos eventos se estrechan lazos de sociabilidad, amparados por la circulacion de discursos e ideas que sellan esos lazos, en busca de una comunión transnacional de carácter emancipador.

Tal como advierten Yankelevich (1997) y Bergel (2015), el "Primer congreso internacional de estudiantes" se lleva a cabo en México, entre el 20 de septiembre y el 8 de octubre de 1921, a instancias del propio Vasconcelos quien, oficiando como presidente honorario, pronuncia el discurso de apertura del evento en nombre de la Universidad. La delegación argentina (integrada por cuantro jóvenes ligados al reformismo: el novecentista Héctor Ripa Alberdi -como presidente-, Arnaldo Orfia Reynal, Pablo Vrillaud y Enrique Dreyzin) alcanza particular visibilidad, lo cual se suma a la importancia inaugural del reformismo en Argentina en general. Estos factores colaboran en la elección de Buenos Aires como sede del siguiente congreso, a realizarse en 1922. Aunque luego este evento no se concreta, el viaje a México deja sus frutos pues a partir de entonces varios intelectuales mexicanos (incluido el propio Vasconcelos) colabora más asiduamente en revistas argentinas ligadas al reformismo. 
El puente abierto en 1921, con el viaje de los jóvenes argentinos a México, se consilida con el viaje a la Argentina de la delegación mexicana presidida por Vasconcelos, en octubre de 1922. Ese viaje oficial, en el marco de un recorrido que anuda sucesivamente el paso por Brasil, Uruguay, Argentina y Chile, entro agosto y noviembre de ese año, se origina en la invitación de parte del gobierno brasilenño, al gobierno de México, para participar en las conmemoraciones del Centenario de la Independencia de ese país, y se prolonga en el convite del gobierno argentino para asistir al cambio de mando de la presidencia, de Hipólito Yrigoyen a Marcelos T. de Alvear. ${ }^{15}$

Luego de ese viaje oficial, Vasconcelos edita su ensayo La raza cósmica. Misión de la raza iberoamericana (1925), reelaborando esa experiencia en base al recuerdo y la imaginación. ${ }^{16}$ Si la primera parte de ese texto constituye una reflexión teórica sobre el problema del mestizaje, la segundo se concentra estrictamente en el periplo sudamericano. ${ }^{17}$ Como veremos, en estas "Notas de viaje" (que guardan sutiles puntos de contacto con la primera parte del libro, titulada "El mestizaje"), el ensayista, además de analizar -a menudo superficialmente. los lugares que visita, también se esfuerza por subrayar su propio liderazgo continental en la defensa de la unidad hispanoamericana y de los movimientos estudiantiles, identificándose como un filósofo mesiánico destinado a relaizar una misión trascendente.

Cuando la delegación mexicana arriba a Buenos Aires, los principales periódicos porteños registran el evento sin dar cuenta de la estatura simbólica de Vasconcelos, más allá de su cargo oficial como representante del gobierno de México. Por ende, es evidente que su valoración como "Maestro de la Juventud" emana más bien de los grupos vinculados a la Reforma Universitaria y al socialismo. ${ }^{18}$ Tal como se percibe en el ensayo, en Buenos Aires Vasconcelos es recibido y acompañado por una multitud de estudiantes reformistas, escandidos por la presencia de Alfredo Palacios, que ven en la política culturar de la Revolución Mexicana una correspondencia con la democratización cultura que promueve la Reforma.

Entre los homenajes que recibe el mexicano en Buenos Aires (y que reconocen en él, a toda una generación de intelectuales revolucionarios), se destaca el acto organizado por la revista Nosotros, en donde José Ingenierons (quein para entonces ya está vinculado con figuras del progresismo mexicano) ${ }^{19}$ pronuncia el discurso "Por la unión latinoamericana", haciendo visible la necesidad de fomentar la integración económica y moral del continente, y la solidaridad con la Revolución Mexicana. En ese discurso (luego difundido en varios medios del continente, y base para la creación de la "Unión Latinoamericana" en 1925), ${ }^{20}$ Ingenieros convierte a Vasconcelos en una especie de catalizador, capas de cohesionar y de multiplicar las "fuerzas morales" del antiimperialismo latinoamericanista. La estadia del mexicano crea nuevos vínculos de solidaridad y profundiza los ya existencies, tal como puede verse en los nuevos viajes y en las declaraciones públicas locales en favor de la Revolución Mexicana y del antiimperialismo por parte de figuras como Alfredo Palacios o José Ingenieros, reforzando la indentificación con al Reforma y ampliando el horizonte de la misma, más allá de los límites universitarios.

Además, tal como se percibe en sus "Notas de viaje", de la lente mexicana e

hispanoamericanista de Vasconcelos emerge una valoración particular de la Argentina, compatible con el enfoque de Ricardo Rojas, al volcarse hacia el interior y el mestizaje indo-hispánico como claves en la definición de la identidad nacional. De hehco, ya en la década del diez, la confrontación entre la perspectiva hegemónica, europeizante e internacionalista y el marginal telurismo de Rojas, se ve cifrada en "Nacionalismo e indianismo", la cara pública que le escribe Ingenieros a Rojas en 1912, descalificando su americanización de la Argentina. En esa polémica interviene Vasconcelos, al adherir a la visión americanizante de Rojas, amén de compartir, con el aurtor de Eurindia, un mismo espiritualismo atnipositivista (en el marco de un esoterismo teosófico común)... junto con la común valoración del mestizaje indo-hispánico como matriz de la identidad continental, la apuesta por una americanización de la Argentina centrada en el interior, y la gravitacion estratégica de Buenos Aires como faro cultural de Amérca Latina. ${ }^{21}$ Cabe aclarar que el espectro ideológico y espistemológico del reformismo argentino resulta por entonces lo suficientemente amplio como 
para permitir el establecimiento de alianzas entre figuras provenientes del positivismo y del antipositivismo, o inclinadas en favor de definiciones antagónicas respecto a la identidad nacional; así, Ingenierons puede legitimar a Vasconcelos, mientras este último aprehende la Argentina desde una perspectiva compatible con la de Rojas (y por ende, opuesta a la de Ingenieros).

Además, el viaje del mexicano ocurre precisamente en el mismo momento en que Rojas (que ha alcanzado una posición privilegiada en la Universidad, en parte gracias a su condición de reformista) ${ }^{22}$ edita su ensayo Eurindia en La Nación. Si bien no se conservan cartas que permitan demostrar el encuentro entre ambos en 1922, ${ }^{23}$ varias figuras importantes (como José Ingenieros, Alfredo Palacios o Alfonso Reyas) operan como nexo entre ellos, al tiempoq ue los dos reciben, en el mismo momento el mote de "Maestros de la Juventud", común entre los principales líderes del reformismo. ${ }^{24}$

Si en el viaje Vasconcelos compara constantemente a la Argentina con México, para reforzar la unidad hispanoamericana, en el discurso que pronuncia en su visita a la Universidad de Córdoba, aproxima los logros culturales de la Revolución a los de la Reforma. En este sentido, en contraste franco con las conferencias académicas de Quesada, las intervenciones de Vasconcelos en el ámbito universitario argentino subrayan los logros culturales de la Revolución Mexicana, y defienden una Universidad políticamente comprometida en la tarea de la democratización cultural, acercando el proceso de transformación mexicano a la Reforma argentina, pensada esta última como el comenzo de un proceso más amplio de inclusión social. Estos tópicos se acumulan en el discurso que pronunica en Córdoba, una ciudad valorada por Vasconcelos como cuna de la Reforma Universitaria y como espacio medélico, po la articulación -a su criterio, privilegiada- del legado hispano-indígena colonial con la experiencia de la modernización contemporánea (que conduce precisamente al movimiento reformista).

Para Vasconcelos, gracias al proceso revolucionario, se expande en México una profunda democratización cultural centrada en la alfabetización, la difusión del conocimiento práctico, la multiplicación de escuelas rurales, la especializacion de la enseñanza estética, la creación de escuelas nocturnas para obreros, el despliegue de la extensión universitaria, el fomento de carreras vinculadas con al modernización del país, la fundación de bibliotecas populares, y la edición de clásicos de la cultura universal, en colecciones de libros baratos, entre otras medidas. Al subrayar estas transformaciones, el mexicano también se auto-legitima a sí mismo como gestor cultural de la Revolución, defendiendo la propia dimensión políticas gracias a resistir la especialización disciplinar y el adacemicismo (que abandera figuras como Quesada, en el seno del mismo movimiento reformista). Pero por sobre todo, ese listado de medidas, precisamente ante el público consagrado por haber dado inico al reformismo continental, insiste en demostrar la convergencia entre Revolución Mexicana y Reforma, subrayando el modo en que en México la Universidad se ha puesto al servicio del pueblo, quegrando el elitismo oligárquico y convirtíendose así el proceso mexicano en un norte capaz de exigir que la reforma desborde los límites de la Universidad: "no hemos nacido del gabinete o de la cátedra [dice Vasconcelos en Córdoba], sino que venimos de la aspiración popular; estamos íntimamente ligados con las necesidades del pueblo [...], y trabajamos tanto con el obrero como con el estudiante" (Vasconcelos 1922: 25) "porque la dirección espiritual la tienen en Méjico los humildes" (Vasconcelos 1922: 28).

Resignificando las categorías conceptuales del arielismo, advierte que México y Argenitna deben asumir la misión trascendente de consolidar la unidad de la "raza hispanoamericana", porque son los países más fuertes y porque, además, ya han superado las luchas internas entre provincias. Y para frenar el peligro del norte, urge difundir el ideal bolivariano de la integración continental.

Así, el discurso que pronuncia en Córdoba condensa implícitamente la diversidad de objetos que convergen en su viaje por Sudamérica: consolidad la unión de Hispanoamérica como freno al imperialismo; difundir la democratización culturar de la Revolución, generando adhesión internacional a este proceso; fomentar la convergencia entre la Revolución y el reformsimo, y reforzar la autoridad del propio Vasconcelos como "Maestro" a nivel continental 
Con respecto a este último aspecto, recreando la iluminación de los discípulos en el final de Ariel de Rodó (aunque ahora el círculo de los "elegidos" es haya amplaido, gracias a la Reforma, hacia todo el estudiantado universitario), el orador corona su exposición instando a los estudiantes de Córdoba, "que han sido líderes de un ilustre movimiento por la liberación del pensamiento de la Argentina", a que "así que conquisten el poder en la Argentina, no olviden todas estas tesis [...] para realizar una unión racional de los países latinoamericanos" (Vasconcelos 1922, p. 41-42). Su expectativa por el desborde de los límites universitarios no puede ser más clara.

No es casual que el viaje diplomático de La raza cósmica (en su doble cariz de representanción política oficial y de magisterio espiritual) se procese y edite en 1925, cuando Vascibcekis ya se encuentra exiliado, 25 porque el texto se hace de la itinerancia del viaje una ventaja ideológica. De hecho, al recordar su visita a Córdoba, reflexiona sobre la importancia de los viajes para la circulación continental de la Reforma, acercado su visita oficial al exilio de los reformistas perseguidos en sus países de origen: estas figuras "se han ido convirtiendo en lazos vivos de unión de las juventudes hispanoamericanas [pues] quizá ellos sean los autores de la gran fraternidad efectiva del futuro" (Vasconcelos 1966, p. 157).

Por eso en La raza cósmica el concepto de "viaje" supone varias modulaciones diferentes, remitiendo tanto al viaje oficial de la comitiva mexicana, como al viaje del exilio (incluso del propio Vasconcelos al momento de editar el ensayo). Es más: así como el término "misión" -convocado en el subtítulo, Misión de la raza iberoamericana- adquiere connotaciones biológicas, políticas y metafísicas en el texto, ese viaje es también, en el marco de las doctrinas esotéricas que sesgan el antipositivismo del ensayista, un desplazamiento simbólico del espíritu. Pues, inscripto en una red que, en esta etapa, adhiere al antipositivismo a partir de un americanismo orientalista, Vasconcelos apela a numerosos elementos esotéricos que redefinen en términos teosóficos el propio concepto de "viaje" en general, y del viaje reformista en particular. Si la segunda parte del ensayo postula que algunos viajes astrales preceden al viaje reformista concreto, la primera sostiene que el mestizaje implica un desplazamiento dialéctico del Espíritu, bajo la forma de una ascensión "racial", biológica y mística al mismo tiempo, que agraga una nueva inflexión trascendente al concepto amplio de "viaje". Y en este punto, la perspectiva de Vasconcelos vuelve a converger con las tesis de Rojas, pues también el argentino concibe el esoterismo como un modelo teórico privilegiado para dar cuenta de la dinámica del mestizaje cultural, pensado como un proceso dialéctico de enriquecimiento "ascensional" del Espíritu. En ambos autores, la gravitación de esta dimensión esotérica del viaje subraya el potencial profético del ensayista como "elegido", y resignifica los lazos materiales de sociabilidad, incluida la solidaridad continental, tan anhelada por algunos autores del reformismo, como parte de una comunión espiritual.

\section{CONSIDERACIONES FINALES}

La figura del "elegido" o del "profeta", en la obra de Vasconcelos, volcado a la tarea mesiánica de consolidad la identidad del contienente, incluso desde la Universidad, contrasta con el ideal de Quesada fundado en el encierro ascético del investigador, que se aparta de la política para defender la autonomía y el academicismo, y que solo interviene en la esfera pública de manera mediada, desde la especificidad de su saber. En este sentido, a inicios de los años veinte, el reformismo cobija definiciones muy polemicas sobre el papel de la Universidad, encarnadas por figuras recíprocamente antagónicas.

Poco después, como en una suerte de calidoscopio ideológico en movimiento, las posiciones de estas figuras vuelve a variar de dirección, modificándose así -en parte al menos- su perspectiva respecto a la Reforma Universitaria, y su vinculo con los jóvenes reformistas, volviéndose más evidentes las tensiones presentes en los discursos de la década previa. Así por ejemplo, Quesada se aleja en 1922 de la Universidad, desalentado por las dificultades para consolidad ese modelo de profesionalización científica, todavía irrealizable en el contexto nacional. Luego de su tangencial indigenismo spengleriano (que alcanza probablemente el clímas político en 
la conferencia dada en La Paz en 1926), Quesada no solo abandona esas hipótesis, sino que además, cuando fija su residencia en la Suiza alemana, estrecha su vínculo intelectual con Spengler, dejando entrever en sus escritos previos a su fallecimiento en 1934, un acompañamiento de la radicalización politica del alemán hacia la ultraderecha. Años decisivos, el libro que edita el alemán en 1933 -en plena consagración del Nazismo-, clausura toda posibilidad de derivar, de la crisis de Occidente, cualquier exaltación indigensita. Por ejemplo, en el último capítulo, "La revolución mundial de color", Spengler advierte que el mundo blanco (Occidente, dominado por el espíritu fáustico) está amenazado por el avance de "los bárbaros de color": África, los indios, los negros y los mulatos de América, al igual que los pueblos islámicos, China y la India, y hasta Java, Japón y Rusia (que es el eje principal de una potencia "asiática")... Para Spengler, estas áreas han acumulado un gran resentimiento por la dominación histórica de los pueblos blancos, y especialmente Rusia es el eje del odio contra Europa blanca; de allí el riesgo real de una resistencia común de todos los pueblos de color de la tierra, en "la victoria del socialismo obrero" (Spengler, 1963, p. 172). Así, el impulso americanista, combinado con el ideario reformista, en los años veinte conduce a Quesasa a legitimar el mundo precolombino y la historia americana, para evaluar un potencial renacimiento indígena; y ese mismo americanismo lo ayuda a Spengler, poco después, a demostrar la amenaza palpable de los pueblos de color. ${ }^{26}$

En el caso de Vasconcelos, cuando regresa a la Argentina como exiliado, en 1933, se revela como una figura francamente reaccionaria, desarmándose por completo el prestigio acumulado en los años veinte, como guía del reformismocontinental. Su giro a la derecha y el desencuentro con el movimiento estudiantil expresan algo de la radicalización política de los años treinta, y la parábola que despliega, en algunos casos, la demanda de acercar Reforma y Revolución Mexicana. Por ejemplo en las tres conferencias que dicta en el Colegio Nacional de La Plata, recién llegoado al país, Vasconcelos presenta la Conquista como una bendición para el progreso del espíritu, y condena la Revolución Mexicana -más allá del rescate aislado de Madero- como un "facundismo" "bolchevizante", dejando entrever su simpatía por el hisanismo católico y por el fascismo. En ese mismo año, los estudiantes de la Federación Universitara Argentina organizn un debate entre Vasconcelos y el pintor comunista David Alfaro Siqueiros, en el que se sella el desprestigo simbólico del viejo Maestro del reformismo continental.

Este giro conservador se traduce en un franco distanciamiento respecto de los intelectuales progresistas argentinos, ${ }^{27}$ y en especial contrasta con el desplazamiento ideológico que por entonces lleva a cabo Rojas, comprometido con la militancia radical en plena dictadura, hasta ser detenido y confinado en el sur (desde donde escribe Archipiélago, para denunciar el exterminio de los indígenas, y reconstruir la cosmovisión de los Onas). Además, en esta etapa, Rojas (que en los años veinte ha sido decano y rector de la UBA), reevalúa la Reforma Universitaria a la luz del radicalismo y el golpe de 1930: en su ensayo El radicalismo de mañana (editado ya en la clandestinidad), inscribe las transformaciones iniciadas con al reforma de 1918 en una teleología ascensional, de resonancias místicas, por medio del cual el pueblo alcanza su "ritmo vital" (Rojas, 1942, p. 263), al que define citando el término sánscrito de sádana.

Exaltando el ideal reformista de una Universidad al servicio de la sociedad, que "siendo costeada por el pueblo, no puede ser una cripta de privilegios doctorales" (Rojas, 1942, p. 270), identifica la Reforma con el radicalismo, y declara que "la Reforma argentina forma trilogía con al reforma electoral y la reforma obrera, porque integra un sistema de modernas instituciones tendientes a quebrar todos los privilegios y a emancipar al pueblo" (Rojas, 1942, p. 271). Incluso, profundizando principios heredados del reformismo, exige la creación de espacios como el "Colegio del Pueblo", para investigar científicamente la realidad argentina y divulgar sus resultados, estudiando las reformas agraria, universitaria, militar, judicial y estética, entre otras, necesarias para "crear un sentido de la vida universal y nacional en la conciencia del pueblo" (Rojas ,1942,p. 271)

En contraste, y acercándose a figuras del nacionalismo de derecha, ${ }^{28}$ Vasconcelos pasa a cuestionar ahora abiertamente al autor de Eurindia, pues "los viejos del liberalismo, aún siendo honrados como Rojas, no se convencen de que es necesario barrer con toda esa patraña de la Revolución Francesa y volver a Notre 
Dame" (Vasconcelos, 1960, p. 313). ${ }^{29}$ Así, tensando su relación con Rojas y con los estudiantes universitarios herederos del reformismo, ${ }^{30}$ Vasconcelos dilapida el capital simbólico acumulando previamente, tanto en su viaje de 1922 como en su ensayo de 1925, como "Maestro de la Juventud" continental. En este sentido, es posible pensar que la radicalización política de los años treinta exacerba las ambivalencias, y las contradicciones que sesgan el reformismo de los años veinte, volviéndolo un espacio de convergencia pero también de disputa.

\section{ReFERENCIAS}

Bergel, M. (2015). El Oriente despazado. Los intelectuales y los orígenes del tercermundismo en la Argentina, Bernal, UNQ.

Buchbinder, P. (2012). Los Quesada, Buenos Aires, Edhasa.

Castillo, H. (1999). Ricardo Rojas, Buenos Aires, Academia Argentina de Letras.

Crespo, R. (2010). “José Vasconcelos, el optimista del ideal: una lectura de La raza

Devés Valdés, E., Melgar Bao, R. (2007). “Redes teosóficas y pensadores (políticos) latinoamericanos”. En Redes intelectuales en América Latina, Santiago de Chile, Universidad de Santiago de Chile, 2007.

Fell, C. (1989). José Vasconcelos: los años del águila (1920-1925), México, UNAM.

Gamio, M. (1960 [1916]). Forjando patria, México, Porrúa.

Ingenieros, J. (1913). “Nacionalismo e indianismo”, Revista de América, año II, vol. II.

Ingenieros, J. (octubre de 1922). “Por la unión latinoamericana” en Nosotros, $\mathrm{n}^{\circ} 161$.

Korn, A. (enero de 1924). "Estudios indostánicos” en Valoraciones, n², La Plata.

Mailhe, A. (2017). "Ricardo Rojas: viaje al interior, la cultura popular y el inconsciente" en Anclajes, La Pampa, Universidad Nacional de La Pampa, ${ }^{\circ} 21$.

Pereira, D. (2008), “Sociología e investigación social en la obra de Ernesto Quesada”, en Políticas de la Memoria, CEDINCI, $n^{\circ} 8 / 9$.

Pita González, A. (2009) La Unión Latinoamericana y el Boletín Renovación. México, El Colegio de México.

Quesada, E. (1895). “La iglesia católica y la cuestión social”, Buenos Aires, Moen.

Quesada, E. (1906). La crisis universitaria, Buenos Aires, Menéndez.

Quesada, E. (1907). “La cuestión obrera y su estudio universitario”, Buenos Aires, Menéndez.

Quesada, E. (1915). “El peligro alemán en Sudamérica”, Buenos Aires, Suárez.

Quesada, E. (1918). "El ideal universitario”, Buenos Aires, Ministerio de Agricultura.

Quesada, E. (1921). La sociología relativista spengleriana, Buenos Aires, Coni.

Quesada, E. (1923). "La faz definitiva de la sociología spengleriana”. En Humanidades, La Plata, UNLP, tomo VII.

Quesada, E. (1923a). "La evolución sociológica del Derecho según la doctrina spengleriana”, Córdoba, Pereyra.

Quesada, E. (1924). “Kant y Spengler”. En Valoraciones, La Plata, t. II.

Quesada, E. (1926). “Spengler en el movimiento intelectual contemporáneo”. En Humanidades, La Plata, Universidad Nacional de La Plata.

Rojas, R. (1951 [1922]). Eurindia, Buenos Aires, Losada.

Rojas, R. (1942). Archipiélago. Tierra del Fuego, Buenos Aires, Losada.

Rojas, R. (1915). La Universidad de Tucumán, Buenos Aires, Librería Argentina de Enrique García.

Rojas, R (1918). Consejo Superior de la UNLP, acta 166.

Rojas, R. (1942 [1932]). El radicalismo de mañana, Buenos Aires, Losada

Spengler, O. (1946 [1918-1922]). La decadencia de Occidente, Madrid, Espasa-Calpe.

Spengler, O. (1963 [1933]). Años decisivos, Madrid, Espasa-Calpe. 
Tarcus, H. (2007). Marx en el 900, Buenos Aires, Siglo XXI.

Vasconcelos, J. (1938 [1920]). Estudios indostánicos, México, Botas.

Vasconcelos, J. (1922). “Orientaciones del pensamiento en Méjico” (folleto), Córdoba, Facultad de Derecho y Ciencias Sociales.

Vasconcelos, J. (1966 [1925]). La raza cósmica. Misión de la raza iberoamericana, México, Espasa-Calpe.

Vasconcelos, J. (1934). Hispanoamérica frente a los nacionalismos agresivos de Europa y Norteamérica, La Plata, UNLP, 1934.

Vasconcelos, J. (1935). Bolivarismo y monroísmo, Santiago de Chile, Ercilla.

Yankelevich, P. (2003). La Revolución Mexicana en América Latina, México, Instituto Mora.

Yankelevich, P. (diciembre de 2006). "El exilio argentino de José Vasconcelos" en Revista Iberoamericana, Madrid, año VI, $n^{\circ} 24$.

\section{Notas}

1 En efecto, un rasgo relevante de su apertura al relativismo cultural se percibe, mucho antes de la recepción de La decadencia..., en sus apuntes de viaje. Luego del viaje a Rusia en 1884 (cuando realiza un recorrido inusual para la época, sobrepasando el límite moderno de San Petesburgo), Quesada publica Un invierno en Rusia (1888); allí establece tempranamente una comparación interesante entre Rusia y América Latina, anticipando parte de la argumentación aplicada luego a Spengler. En efecto, en ese libro, además de reclamar la introducción urgente de reformas, para modernizar el imperio y evitar el advenimiento de una revolución, subraya el paralelo entre los pueblos ruso y americano, porque en ambos el carácter multicultural define un recorrido hacia el progreso diverso respecto del seguido por Europa (incluso, Quesada compara a Rusia con Argentina, por la gran extensión territorial, la poca densidad de población, la fractura entre los mundos urbano y rural, y las dificultades para la afirmación de la identidad nacional, entre otros rasgos). Y en su libro Una vuelta al mundo (1913), resultado de otro largo viaje, revela su temprana apertura al relativismo cultural, porque allí se detiene exclusivamente en destinos exóticos como Argelia, Túnez, Egipto, Palestina, India, Ceilán, Filipinas, China, Corea y Japón.

2 El discurso que da en 1921, al cumplirse el primer centenario de la UBA (editado en su curso de 1921 sobre Spengler) es un buen indicador de adhesión a la Reforma Universitaria, así como lo es su conferencia "el ideal universitario" (1918), o su nombramiento como interventor de la Facultad de Derecho de la UBA.

3 Así por ejemplo en su conferencia "la cuestión obrera y su estudio universitario" (1907) insiste, antes nuevas huelgas, en que el Estado estudie la "cuestión social" con nuevos métodos científicos, para que el problema social no sea apropiado por los marxistas. Ese reformismo también se expresa en " La iglesia y la cuestión social" (1985): allí Quesada plantea la urgencia por atender la cuestión social, justamente para evitar el estallido de una revolución socialista. Quesada rechaza el colectivo marxista, y solo cuestiona al capitalismo laissez-faire, pidiendo que el Estado y la iglesia católica aunen fuerzas para evitar un estallido social. Así, en 1907, insiste en subrayar la importancia del Estado. Esto se ve tanto en sus estudios universitarios como en su gestión en el Departamento de Trabajo. Al respecto ver Tarcus (2007).

$4 \mathrm{Al}$ respecto ver su conferencia con motivo del centenario de la UBA, el 12 de agosto de 1921 en Quesada (1921 pp 595 y ss.). Allí Quesada se distancia fuertemente de la especulación financiera, e insiste en la necesidad que las clases dirigentes inviertan en las universidades, convirtiendo la plutocracia en ilustración. (Quesada, 1921, p. 608).

5 Sobre los neokantianos locales ver Dotti (1992).

6 Según Carreras (2008). Quesada lee el primer tomo del libro de Spengler gracias al regalo de navidad enviado a Deiters desde Alemania.

7 Carreras (2008) recuerda que la relación entre Quesada y Deiters comienza en 1913, cuando la segunda entrevista a Vicente Quesada en Buenos Aires, como corresponsal de un periódico alemán. Luego Deiters le pide a Quesada notas en defensa de Alemania, cuando se inicia la "Gran Guerra", para editarlos en el periódico en el cual trabaja, el Kölnische Zeitung (y a cambio, difunde en ese medio la obra de Quesada). En 1915, el vinculo intelectual se convierte en amoroso: finalizada la guerra Deiters viaja a Buenos Aires para unirse a Quesada, con quien viajan por Europa y América Latina. Ambos trabajan en una estrecha colaboración intelectual (favorecida, entre otros elementos, por la pertenencia común a la burguesía culta y cosmopolita de sus respectivos paises).

8 Ver Quesada (1923)

9 El curso se centra solo en el primer tomo de La decadencia..., el único editado para 1921. En total Quesada dicta 44 clases (integradas en el libro), 30 de exposición de la teoría spengleriana y 14 de crítica general de la obra.

10 Ver Pereira (2008) 
11 Además. Quesada asocia la perspectiva relativista de Spengler a la de Hernan Keyserling (en particular cita al libro de viajes -que dice que ha tenido gran impacto en Alemania- titulado Das Reisetagebuch eines Philosophen, de 1920)

12 Incluso, Quesada se reprocha a sí mismo el hecho de no haber formado a los estudiantes de ese año en arqueología americana. Además, respondiendo a la demanda de bibliografía específica, por parte de Spengler, traza un mapa de las principales publicaciones sobre el tema editadas en Francia e Inglaterra.

13 Quesada defiende el intuicionismo spengleriano contra el neokantismo dominante en un amplio sector del antipositivismo reformista local. Ver por ejemplo Quesada (1924), editado en la revista Valoraciones (sobre la adhesión de esta publicación periódica al reformismo, es posible consultar el trabajo de Galfione, en este mismo dossier)

14 Se trata de un cargo semejante al de Ministro de educación, en Argentina.

15 El gobierno de Alvaro Obregón ve en el convite la oportunidad de profundizar sus relaciones con el subcontinente, por lo que envía una delegación de 400 personas, presididas por Vasconcelos como Embajador especial.

16 Vasconcelos dice no llevar un diario de viajes, sino confiar en que "el alma" pueda recordar lo más importante. Esa advertencia no es menor, dado que el ensayista da rienda suelta al impresionismo, tan característico de su escritura.

17 Tal como señala Fell (1989), la primera versión de "Notas de viaje" tiene más de 200 páginas por ensima de la versión que publica luego Espasa-Calpe en 1948: Aquí Vasconcelos hace silencio sobre su visita a Uruguay y a Chile, y retira pasajes completos (como su visita a Leopoldo Lugones), para no contradecir su tesis sobre el destino utópico de América Latina.

18 Por ejemplo, La Nación registra, el 6 de octubre, la llegada del Ministro de México al puerto de Buenos Aires, sin referencias específicas a Vasconcelos, y el 11 de octubre Vasconcelos es apenas nombrado entre las numerosas personalidades que asisten al desfile oficial y a la entrevista personal con el presidente saliente. Tampoco hay registros en este medio del viaje de Vasconcelos por el país.

19 Yankelevich (2003) señala que por entonces Ingenieros ya es conocido en México, especialmente en sectores revolucionarios, al tiempo que fomenta la unidad latinoamericana y el antiimperialismo, desde una perspectiva afin a los intereses de la Revolución Mexicana.

20 Por ejemplo, es publicado por la revista costaricense Repertorio americano ( $\mathrm{n}^{\circ} 18$, enero de 1923)

21 Sobre este tema en Rojas ver Mailhe (2017).

22 Rojas es reconocido como un ideólogo del reformismo incluso antes de 1918. Este reconocimiento colabora en su elección como Decano de Filosofía y Letras de la UBA, con voto de alumnos y profesores en 1922, y como Rector de la misma universidad en 1926.

23 En la Casa Museo de Rojas, no se conservan cartas enviadas por Vasconcelos a Rojas. En la biblioteca del autor de Eurindia apenas hay dos obras del mexicano, con dedicatorias al "Maestro" Rojas. En cambio, la correspondencia del ateneísta Alfonso Reyes es nutrida, poniendo en evidencia no solo el vínculo de mutua colabración, sino también el reconocimiento de Rojas en el ambiente revolucionario mexicano.

24 Como ejemplo de la identificación de ambos intelectuales como líderes de la juventud, en el archivo personal de Rojas se conserva una nota, guardada por él, aparecida en "El Comercio de Lima" el 16 de agosto de 1923, en donde Rojas (como Rector de la UBA) y Vasconcelos (a cargo de la Secretaría de Educación Pública) son presentados como los "principales directores del pensamiento de América". Las referencias al caracter de "Maestro" proliferan entre los líderes del reformismo (por ejemplo Vasconcelos elogia a Palacios como "Maestro de la Juventud" y "Apostol argentino del iberoamericanismo"; Vasconcelos 1966, p. 165).

25 En 1924 Vasconcelos renuncia a la Secretaría de Educación Pública, por disidencias políticas. A partir de aquí se abre una etapa de declinación, marcada por la elaboración de resentimiento creciente contra México. Cuando es derrotado en las elecciones de 1929, inicia un largo exilio de casi 10 años en EE.UU., Centroamérica, Colombia, Cuba, Ecuador, Brasil, España, Francia y Argentina, para volver finalmente a EE.UU.

26 El argumento de Spengler remata entonces con una consagración de Alemania, más explícita que en La decadencia...: el pueblo alemán es el menos decadente de la raza blanca, pues en él se conserva el alma fáustica, clave en la civilización occidental; además, de todo Occidente, el pueblo alemán es el único que no ha pasado por el desgaste de ser una potencia imperialista; por ende, es el que debe tomar el poder, a través de un gobierno fuerte, para evitar el avance de otros pueblos que estan despertando como Rusia, Japón y las masas latinoamericanas.

27 En cambio, Palacios se esfuerza por defenderlo. Por ejemplo, en el prólogo a Hispanoamérica..., construye una imagen insostenible de Vasconcelos como un peregrino idealista, "un auténtico Maestro de la Juventud" que se sacrifica por el continente, pero que es desoído por los jovenes y advierte que "sería lamentable que [...] le dejaran pasar con indiferencias o con enojo" (Palacios, 1934: 100).

28 Incluida una entrevista con el Presidente de facto Agustín P. Justo.

29 En la biblioteca personal de Rojas, conservada en su Casa Museo, existen apenas dos libros de Vasconcelós, ambos con dedicatorias personales que evidencias cierto vínculo de amistad respetuosa de parte del mexicano, y ambos correspondientes a la estapa de exilio de Vasconcelos en Argentina o a su salida del país: el folleto Hispanoamérica frente a los nacionalimos agresivos de Europa y Norteamérica (1933), dedicado al "Maestro Rojas, con la admiración y aprecio 
de José Vasconcelos", y el libro Estetica (1936) que reza, "para Don Ricardo Rojas, con el viejo afecto admiratico de su amigo José Vasconcelos".

30 Tal como reseña Yankelevich (2006), el debate con Siqueiros y las propias intervenciones públicas de Vasconcelos en general ponen en evidencia la rapidez con la que el segundo pierde su popularidad como lider del reformismo a nivel continental. 